Claudia Colini, Ivan Shevchuk, Kyle Ann Huskin, Ira Rabin, Oliver Hahn

\title{
A New Standard Protocol for Identification of Writing Media
}

\begin{abstract}
Our standard protocol for the characterisation of writing materials within advanced manuscript studies has been successfully used to investigate manuscripts written with a pure ink on a homogeneous writing surface. However, this protocol is inadequate for analysing documents penned in mixed inks. We present here the advantages and limitations of the improved version of the protocol, which now includes imaging further into the infrared region (1100-1700 nm).
\end{abstract}

\section{Introduction}

To assist scholars of manuscript studies, the Federal Institute of the Materials Research and Testing (BAM) in Berlin and the Centre for the Study of Manuscript Cultures (CSMC) in Hamburg have developed a two-part protocol of nondestructive ink analysis: a primary screening performed with NIR (near infrared) and UV (ultraviolet) reflectography at two specific wavelengths, followed by an in-depth analysis using spectroscopic techniques that include X-ray Fluorescence (XRF) and, in individual cases, Fourier Transform Infrared (FTIR) and Raman spectroscopy. ${ }^{1}$ The primary screening was specifically developed for manuscripts written on homogeneous substrates with a pure ink type. ${ }^{2}$ The inadequacy of the initial protocol became increasingly apparent during the studies of papyri, manuscripts coming from an archaeological contest, and Arabic inks. ${ }^{3}$

1 Rabin et al. 2012.

2 Rabin 2015; Cohen et al. 2017.

3 We are referring in particular to $\mathrm{PhD}$ researches on Coptic manuscripts written on papyrus and parchment and dating from the second to the eight centuries CE (Ghigo 2020 and Ghigo et al. 2020); on Arabic-Jewish manuscripts from the Cairo Genizah written on paper and parchment in the eleventh century CE (Cohen 2020); and on black inks reproduced from Arabic recipes dated from the ninth to the twentieth centuries CE (Colini 2018). 
When organising a new research project to focus on the materiality of Arabic fragments from Egypt during the first five centuries of Islam, it was clear from the historical material circumstances that the standard protocol would need an update. ${ }^{4}$

During the Umayyad period, and specifically at the beginning of the eighth century CE, when the Arabs conquered Central Asia, paper was introduced into the Islamic world thanks to the Sogdians, who were familiar with both Chinese and Central Asian paper types. ${ }^{5}$ The new writing material was positively received, in particular the Central Asia type obtained from rags, and it eventually replaced papyrus, which was the main writing support, together with parchment, until the tenth century CE. In Egypt, the transition from papyrus to paper took place between 870 and $970 \mathrm{CE}$, although paper had already become the preferred writing support by 930-940 $\mathrm{CE}^{6}{ }^{6}$ A combination of economic advantages and of prestige and legitimacy embedded in the new writing material seems to have been the reasons motivating this transition. ${ }^{7}$

Parchment was also frequently used, in Egypt and in the Islamic world, but it was specifically employed for relevant and durable contracts (such as purchase deeds and documents related to marriage), luxurious items and texts connected with religion and esoterism (Qur'ans and Jewish liturgical scrolls, for instance, but also amulets and magical texts). ${ }^{8}$ Parchment continued to be used after the introduction of paper, and although it was never completely substituted by the new writing support, its use slowly decreased in the following

\footnotetext{
4 The research project RFA01 'The Scribe's Choice: Writing Supports in Arabic Documents of the Early Islamic Centuries', part of the Excellence Cluster 2176 'Understanding Written Artefacts', Universität Hamburg.

5 The earliest surviving Arabic texts written on paper are the documents found at Sanjar-Shah (Tajikistan) and dating between 720 and 790 CE. Their paper was most likely imported from China, as it is made of pure new paper mulberry fibres, and differs from the Central Asian type as this is made of recycled fibres (mainly hemp, ramie and flax) from rags; Haim et al. 2016, 163-168; Shatzmiller 2018, 472-475. For a detailed explanation concerning the diffusion of paper by Sogdians and the adoption by Umayyads and Abbasids, see Rustow 2020, 116-135.

6 Grob 2010, 11-13; Rustow 2020, 135. It is possible, however, that paper was imported, mainly from Syria, as early as 860 CE; Youssef-Grob 2015, 431-432.

7 See Shatzmiller for the economic reasons (Shatzmiller 2018, 475-485) and Rustow for the prestige associated to paper (Rustow 2020, 135-137).

8 Rustow 2020, 137; Cohen 2020, 92-94; Bausi et al. 2015, 89-90. Entries of parchment fragments in the Arabic Papyrology Database (APD) confirm this trend <https://www.apd.gwi.unimuenchen.de/apd/show_new.jsp> (accessed on 10 Dec. 2020).
} 
centuries. Less common writing supports used in Egypt include leather, textile, bones, wooden tablets and ostraca. ${ }^{9}$

With regards to writing media, an overall familiarity with inks of different types and chemical compositions is well attested in the Middle East in the tenth and eleventh centuries CE. The inks of that time belonged to three typologically distinct classes: carbon, plant, and iron-gall inks. ${ }^{10}$

Carbon inks are obtained by mixing charcoal or soot with a water-soluble binder, such as gum arabic (common in the Middle East) or proteinaceous glues (common in the Far East), and dispersing the mixture in a water-based solvent. ${ }^{11}$ Plant inks are mainly composed of tannins and are obtained by cooking or macerating various vegetal materials. Unlike the plant inks used in Europe, gall nuts are rarely used as ingredients according to the Arabic sources; instead, the few Arabic recipes call for bark, fruits or flowers. ${ }^{12}$ Iron-gall inks are obtained by the reaction between iron ions $\left(\mathrm{Fe}^{2+}\right)$ with gallic acid in a water-based solvent, with the addition of a binder, normally gum arabic. The most common source of iron ions is vitriol (a mixture of hydrated metallic sulphates), but the recipes also attest to non-vitriolic iron-gall inks, which were prepared using filings, slags, nails and pieces of iron. Though the best source of gallic acid is gall nuts, they could be substituted by a variety of tannin-rich plant matter (e.g. tree bark, fruits and fruit rinds, leaves, etc.) that was cooked or macerated.

The distinct optical properties of carbon, plant and iron-gall inks can be used to differentiate them: carbon ink maintains its solid black colour across the ultraviolet, visible and infrared regions of the light spectrum; plant ink turns transparent at around $750 \mathrm{~nm}$; and iron-gall ink gradually loses opacity towards the longer wavelengths. ${ }^{13}$ Furthermore, tannins' ability to quench fluorescence enhances the contrast between a fluorescing background and the text, making UV reflectography a useful tool for identifying their presence.

In addition to observing their distinct optical properties, quantitative and semi-quantitative X-ray fluorescence spectrometry (XRF) measurements can also be used to detect differences in the elemental composition of marker elements (such as copper, manganese or zinc in vitriol-based iron-gall inks), making this another powerful tool for manuscript studies. The fingerprint model,

9 Grohmann 1952, 44-62.

10 Cohen et al. 2017; Rabin 2017; Colini 2018, 17-18 and 39-40.

11 Zerdoun 1983, 55-67 and 71-90.

12 For European recipes of plant inks made with the extract of gall nuts, see Zerdoun 1983, 180 and 306-307. For Arabic plant inks, see Colini 2018, 40 and 119-220.

13 Mrusek et al. 1995, 72. 
introduced some fifteen years ago, presents iron-gall ink as a series of ratios of the vitriolic impurities to iron (the main component of the ink) and also considers the relevant impurities of the paper..$^{14}$ The use of NIR imaging, accompanied by a semi-quantitative calculation of the iron-gall fingerprints, have shown excellent results in advanced codicological studies of medieval European manuscripts on paper and parchment. ${ }^{15}$

Besides these pure types, another class of inks appears to have been quite popular among the Arabic sources: mixed inks. ${ }^{16}$ Depending on how these inks were prepared, two subtypes can be defined: mixed carbon-plant inks and mixed carbon-iron-gall inks. The former, which are characterised by the addition of plant extracts rich in tannins to a carbon ink, are attested in the sources as early as the ninth century $\mathrm{CE}^{17}{ }^{17}$ Recipes of mixed carbon-iron-gall inks appeared later, in the thirteen century, although one of them is attributed to a vizier who died in $806 \mathrm{CE} .^{18}$ Their formulations can range from adding a small amount of soot or charcoal to an iron-gall ink, to adding vitriol and tannins to a carbon ink; therefore, inks with different ratios of carbon to iron are necessarily included in the same subtype. Currently, use of the mixed inks in Medieval period are considered rare and are only sporadically identified in manuscripts; however, this may be due to the difficulty of their identification rather than their absence. ${ }^{19}$ Rabin suggests that mixed inks played an important role in the transition from the carbon inks of Antiquity to the iron-gall inks of the Middle Ages. ${ }^{20}$

Currently, a growing number of publications report presence of metals in the inks dating to the Greco-Roman period: carbon-based inks that also contain different admixtures or metallic elements, such as copper and lead..$^{21}$ Christiansen attributes presence of copper in the inks to the use of soot that came from metallurgical plants, while lead compounds could be used for their drying

14 Hahn et al. 2004.

15 Hahn et al. 2008a; Geissbühler et al. 2018.

16 Colini 2018, 17-19 and 40.

17 Colini 2018, 39. The recipes can be found in the appendix, Colini 2018, 119-220.

18 Colini 2018, 40. The recipes can be found in the appendix, Colini 2018, 119-220.

19 The limitation of the current protocol for identification of the tannins is discussed in Rabin 2015, Colini et al. 2018, Cohen 2020, Ghigo et al. 2020, Ghigo 2020.

20 Rabin 2017, 7-11, Nehring et al. 2021.

21 Carbon inks containing copper were found in the Genesis Apocryphon scroll (1Q GenAp) from the Dead Sea Scrolls collection (Nir-El and Broshi 1996), in two fragments of private letters from Pathyris and in two others from the Tebtunis temple library (Christiansen et al. 2017), and a documentary papyrus from Fayum (Rabin et al. 2019). Carbon inks containing lead were identified in two fragments of the Herculaneum papyri (Brun et al. 2016), a fragment from Dime (Colini et al. 2018) and two others from the Tebtunis temple library (Christiansen et al. 2020). 
properties.22 It must be stressed, however, that in the absence of a nondestructive method that can unequivocally identify the presence of tannins in the ink, it is impossible to differentiate between a carbon ink with an admixture of iron or copper from a carbon-iron-gall mixed ink.

Here, we present the tests conducted with the new mobile equipment OPUS Instruments APOLLO Infrared Reflectography Imaging System (IRR), intended to unambiguously identify carbon in mixed inks. During the preparatory stage of the aforementioned research project, fifteen Arabic fragments preserved in the Department of Papyri of the Austrian National Library (ÖNB), Vienna, were tested.

\section{Description of the corpus}

Table 1 summarises the known historical data of the fifteen Arabic fragments from the Department of Papyri of the Austrian National Library (ÖNB), Vienna, investigated in this work. The collection was established in 1883 following several purchases made between 1878 and 1882 by the Archduke Rainer who was persuaded by Josef von Karabacek (1845-1918), Professor of History of the Orient at the University of Vienna, to buy a large number of papyri from the Viennese antiques dealer Theodor Graf (1840-1903). ${ }^{23}$ The majority of the fragments in the collection come from the excavation sites of al-Ušmūnayn (the ancient city of Hermopolis Magna) and Ihnasiya (Heracleopolis Magna) in central Egypt, as well as various archaeological sites in the Fayum oasis. ${ }^{24}$

22 The first statement can be found in Christiansen et al. 2017, 7; the second in Christiansen et al. 2020, 27834.

23 <https://www.onb.ac.at/en/library/departments/papyri/about-the-department-of-papyri> (access on 06 Apr. 2021).

24 When talking about provenance, a distinction should be made between the finding place, the actual place of production of the written artefacts and the internal references to locations in the texts. Since the fragments in the collection were acquired through the antiques market, the place of production is extremely difficult to identify, and while the finding place is often provided in the acquisition records, they are not always reliable. Finding place and area of production may coincide, but this is not always the case: letters, for example, often have a different place of production than the site where they were excavated. When a location mentioned in the text, such as a village referred to in a sales contract, can be found, it is possible that this location coincides with the place of production, although each instance should be evaluated individually. In this paper we are using the concept of provenance in a broad sense, including all the aforementioned meanings. 


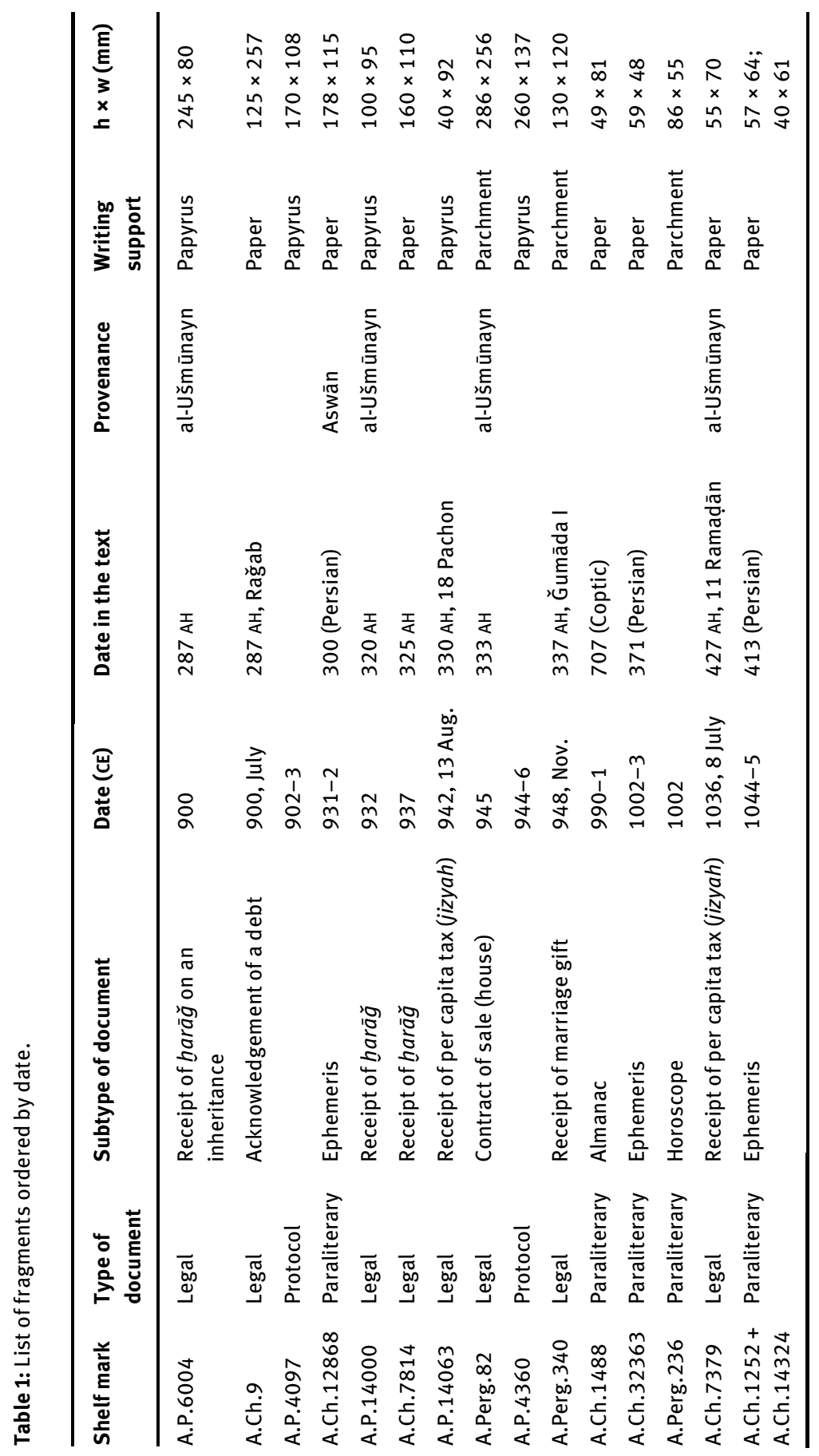


The manuscripts selected for this study - written in black inks on parchment, papyrus and paper - are dated between the tenth and the first half of the eleven century CE and represent different contents and contexts of production.

\section{Experimental section}

The writing supports of the fragments were first described according to their visual characteristics, both in ambient and transmitted light (using a light sheet). The latter is commonly used to observe the marks left by the sieve during paper production, but it also reveals the fibre distribution of a papyrus sheet.

\subsection{Dino-Lite USB microscope, AD413T-I2V}

Following a visual description, we conducted the primary screening with a handheld Dino-Lite USB microscope. Measuring just $10 \mathrm{~cm}$ in length, this miniature microscope features built-in LED illumination at $395 \mathrm{~nm}$ (UV) and $940 \mathrm{~nm}$ (NIR), as well as a customised external white light (VIS) source mounted on the microscope. This instrument is used to differentiate the types of inks by comparing the change of opacity between the visible and the infrared image. In addition to the NIR micrographs, we usually also examine the images captured under UV light: the presence of tannins can be inferred from the enhanced contrast of ink and substrate, a result of tannins' ability to quench the UVinduced fluorescence of organic supports. In some cases, the dimensions of the writing stroke appear larger in the UV image compared to the VIS image due to the spreading of tannins in the writing support.

\subsection{Elio Bruker Nano GmbH (formerly XG Lab)}

This X-ray spectrometer features a $4 \mathrm{~W}$ low-power rhodium tube and adjustable excitation parameters. It has a $17 \mathrm{~mm}^{2}$ Silicon Drift Detector (SDD) with energy resolution < $140 \mathrm{eV}$ for $\mathrm{Mn} \mathrm{K \alpha}$. The beam size is roughly $1 \mathrm{~mm}$. The measurements were performed on single spots at $40 \mathrm{kV}$ and $80 \mu \mathrm{A}$, with an acquisition time of 2 minutes. Bruker's SPEKTRA software was used for the peak fitting and the semi-quantitative data evaluation. This simple portable instrument is usually the first choice when a quick identification of elemental composition is required and the inked areas are compatible with the beam spot size. 


\subsection{OPUS Instruments Apollo}

Verification of the presence of carbon in the inks was performed with OPUS Instruments APOLLO Infrared Reflectography Imaging System (IRR). The regular Short-Wave Infrared (SWIR) sensing range $(900-1700 \mathrm{~nm}$ ) of the $128 \times 128$ pixel scanning InGaAs sensor was reduced by the following filters: Short Wave Pass Filter (SWP1250, range 900-1250 nm), Band Pass Filter (BPF1250-1510, range 1250-1510 nm) and Long Wave Pass Filter (LWP1510, range 1510-1700 $\mathrm{nm})$. Each filter was mounted in front of the IR lens $(150 \mathrm{~mm}, \mathrm{f} / 5.6-\mathrm{f} / 45)$ with the aperture set to $\mathrm{f} / 11$. The working distance between the sensor and the object was set to $73 \mathrm{~cm}$ and the acquisition time to $50 \mathrm{~ms}$ per tile. Two $20 \mathrm{~W}$ halogen lamps provided broadband illumination. The filters allow us to limit the range of infrared light to the portion where iron-gall inks become completely transparent, thereby enabling the discrimination of pure iron-gall inks and mixed inks.

\section{Results and discussion}

The fact that the original protocol was never intended for the complete characterisation of mixed inks, which were hardly employed in late medieval or European manuscripts, has been discussed at length elsewhere. ${ }^{25}$ Here, we would like to present three selected examples from the corpus to illustrate some common classes of inks encountered during field studies. Fig. 1 shows, on the right, the photographs of these fragments; the blue squares highlight the area shown in the Dino-Lite micrographs, taken in visible light and at $940 \mathrm{~nm}$ (NIR), which appear in the middle of the figure. On the left, we present the XRF spectra of the writing supports and of the inks.

The ink of the papyrus fragment A.P.4360, in Fig. 1a, is easily identified as an iron-gall ink because its opacity decreases noticeably (but not completely) at $940 \mathrm{~nm}$ when compared to the visible light image. Furthermore, in the XRF spectra, the signal of iron from the inscribed area far exceeds that detected on the uninscribed papyrus. The ink of the main text of the contract on the parchment manuscript A.Perg.82, in Fig. 1b, is most probably of the carbon ink variety, as there is neither a change in opacity at $940 \mathrm{~nm}$ nor any meaningful change between the spectra of the inscribed and uninscribed areas.

25 Colini et al. 2018; Ghigo et al. 2020. 

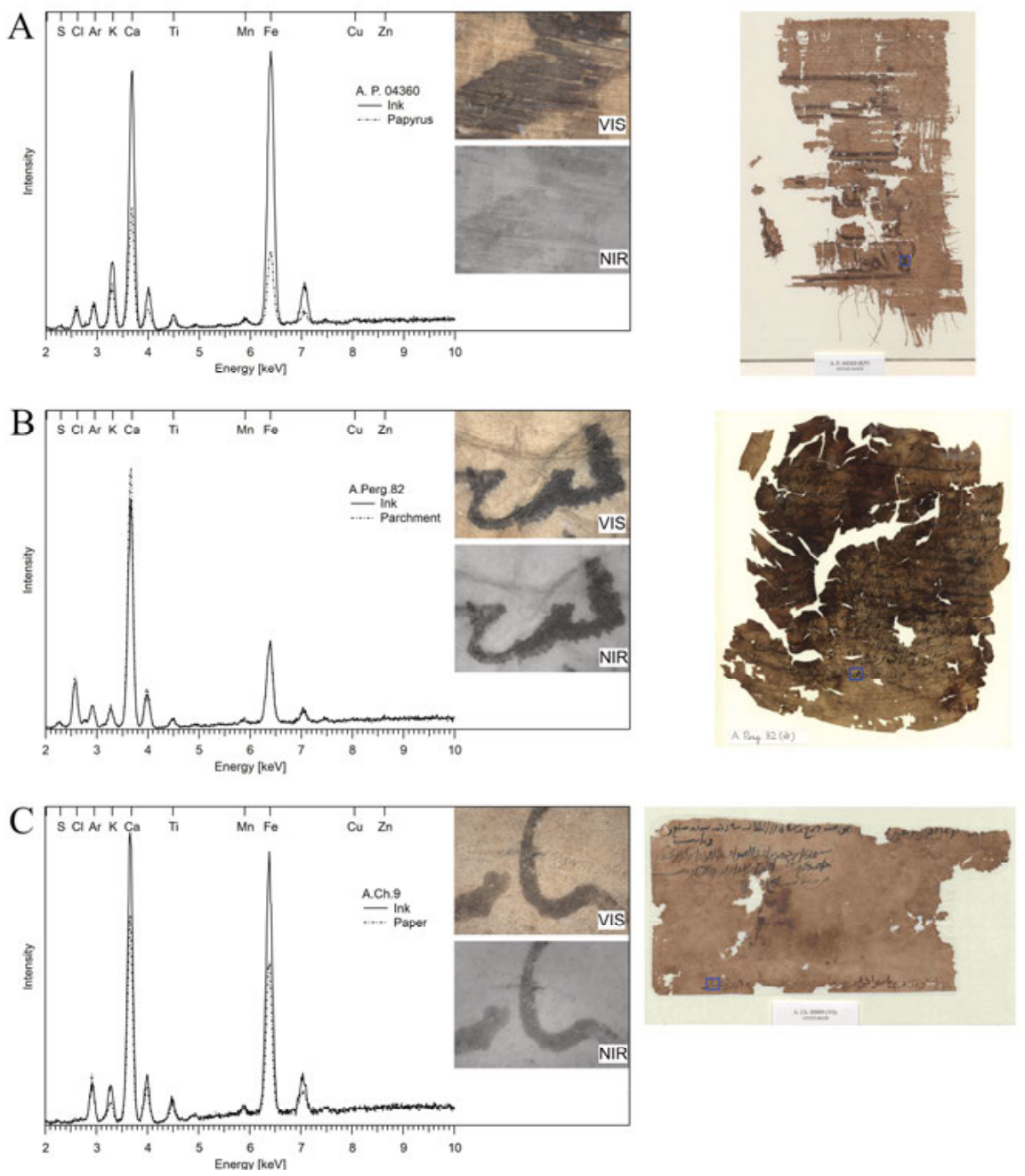

Fig. 1: On the left, XRF spectra of writing supports and of inks; micrographs at 50x magnification of the measured spot taken with Dino-Lite in VIS light and at $940 \mathrm{~nm}$ (NIR); on the right, image in visible light of the entire fragment with the area of the micrographs highlighted in blue: A) A.P.4360; B) A.Perg.82; C) A.Ch.9. Manuscript images (־ Österreichische Nationalbibliothek, Papyrussammlung.

The fragment in Fig. 1c, A.Ch.9, contains four different inks; the ink in question is found at the end of the bottom line, a later remark attesting that debt has been partially cleared. This line is written with two inks, the beginning with a brown-red one that has corroded the paper, the ending with a darker one and 
slightly thinner pen strokes. ${ }^{26}$ The ink in which these last words were written shows little opacity change at $940 \mathrm{~nm}$ compared to the visible image, as seen in the Dino-Lite micrograph in Fig. 1c. By comparing the XRF spectra of this ink and the writing support, an increase in the amounts of iron, calcium and potassium in the inscribed area can be detected (black curve). Based on the current protocol, we tentatively conclude that we are dealing here, in A.Ch.9, with a non-vitriolic carbon-iron-gall mixed ink.

As the case in Fig. 1c illustrates, it is difficult to verify the presence of iron in the writing media because the paper also contains a high amount of the same element. This is indicative of a larger problem of identifying mixed inks, on papyrus or Arabic paper using the XRF-method. The low iron signal in the ink, accompanied by a heterogeneous distribution of iron in the writing support resulting in a strong noise of the iron signals, makes the use of the fingerprint model practically impossible, since the calculations require small quantities and homogeneous distribution of iron signals in the writing support.

In Fig. 2, we illustrate the average iron content of three fragments (A.Perg.82, A.Ch.32363 and A.P.14063) from the Vienna Papyri Collection. The error bars represent the standard deviation (i.e. the dispersion of the data relative to its arithmetical average).

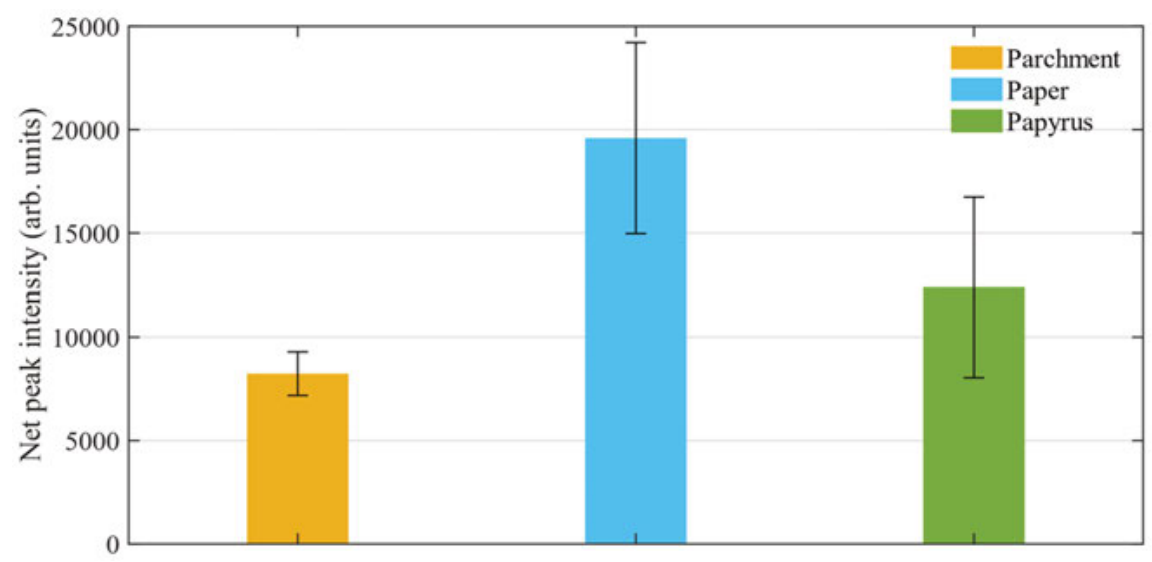

Fig. 2: Distribution of iron in the writing supports of three fragments: A.Perg.82 (parchment, in orange), A.Ch.32363 (paper, in light blue) and A.P.14063 (papyrus, in green).

26 Unfortunately, the edition of the fragment does not clarify whether these last words were added to the line or if they were written by another scribe. In fact, this part of the document could only partially be read and translated due to its preservation state; Youssef-Grob 2015, 440-441. 
As we can see, the writing supports of the analysed fragments have high iron concentrations, especially paper and papyrus. The smaller error bar of the parchment average, compared to those of paper and papyrus, indicates that the distribution of iron in the parchment fragment is more homogeneous. The heterogeneous distribution of elements is caused by the intrinsically uneven structure of papyrus, and also by residual archaeological materials (e.g. dirt, sand, etc.) that contaminate it with iron. It is possible that the tools used to cut and beat the rags as well as the water employed in papermaking were responsible for the iron contamination in paper fragments. Furthermore, conservation treatments or abrasions resulting from past use may also contribute to the contamination of the writing support or the wider distribution of iron that was initially localised in the inked area. ${ }^{27}$ Thus, not only do paper and papyrus contain more iron, but its distribution is also extremely uneven. ${ }^{28}$

It is clear that when NIR reflectography at $940 \mathrm{~nm}$, coupled with XRF, fails to identify the ink type, we must resort to the IR reflectography method originally proposed by Mrusek and colleagues. ${ }^{29}$ In this study, we tested the Infrared Reflectography Imaging System (IRR) OPUS Instruments Apollo, in addition to the NIR screening conducted with the Dino-Lite and XRF analysis conducted with Elio.

The performance and suitability of the instrument were tested on a set of three-year-old ink samples prepared at the manuscript lab of the CSMC, University of Hamburg. ${ }^{30}$ Fig. 3a shows a picture of the inks - respectively, an iron-gall ink, a carbon ink, a mixed carbon-plant ink and a mixed carbon-iron-gall ink taken in visible light. The images in Fig. 3b-c-d were taken with the Apollo imaging system equipped with the SWP1250, BPF1250-1510 and LWP1510 filters, respectively. As expected, the carbon ink shows no change in opacity across all four images, while the mixed inks progressively become more transparent, although they remain visible even in Fig. 3d. The iron-gall ink, in contrast, shows

27 Water-based conservation treatments can cause the delocalisation of the soluble parts of the iron-gall inks resulting in the emergence of an iron-rich background in the writing support; Hahn et al. 2008b. The use of the tannic tincture caused delocalization of iron and potassium in the Vercelli Book; Rabin et al. 2015.

28 Similar observations based on a higher number of documents can be found in Cohen 2020, $120-124$ and 142-150.

29 Mrusek et al. 1995.

30 The inks were prepared in 2016 according to Arabic recipes. They were artificially aged for 49 days in the ageing chamber WK11-180/40 by Weiss Umwelttechnik GmbH at BAM, with the condition of $\mathrm{T}=80^{\circ} \mathrm{C} ; \mathrm{RH}=65 \%$, and subsequently stored in the rooms of CSMC. Colini 2018, 5978 (nos $17,52,165,173)$ and $82-87$. 
a clear change in opacity in Fig. 3b, while traces of it are barely detectable in Fig. 3c, and it becomes completely undetectable in Fig. 3d.
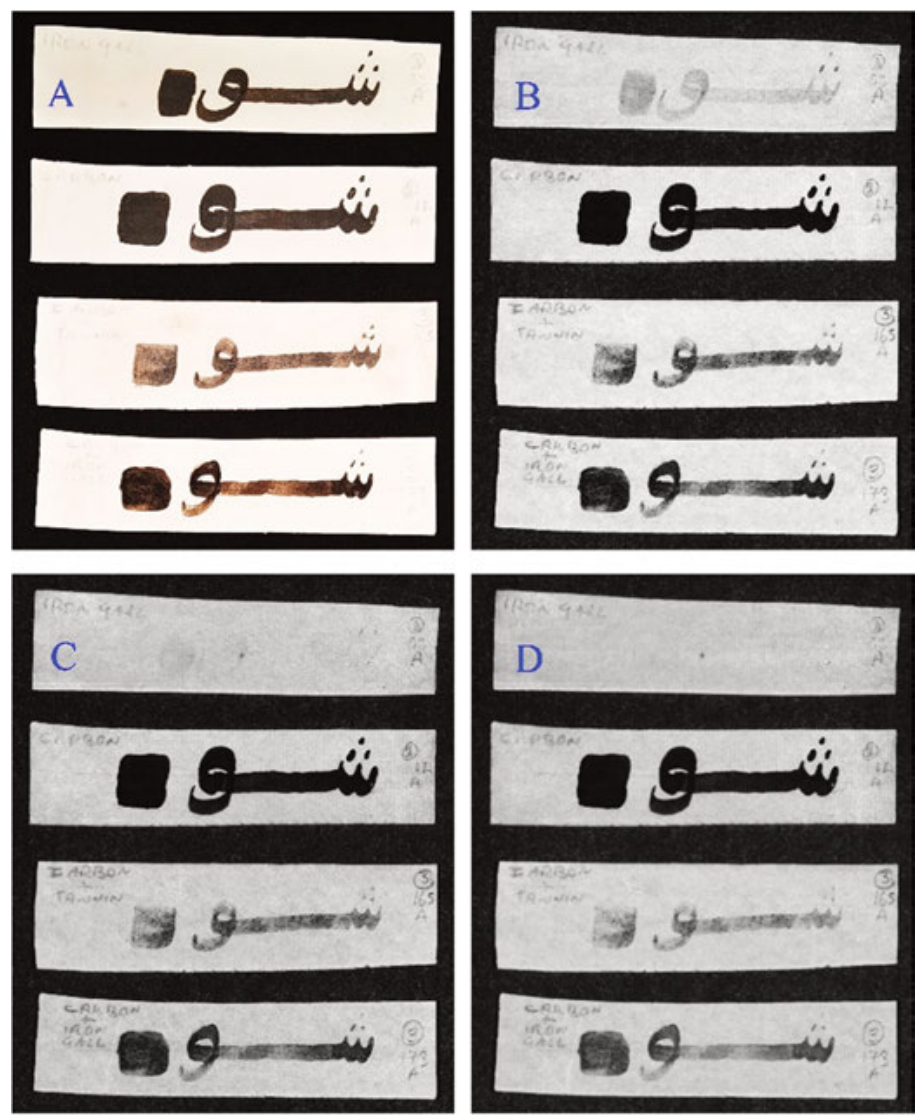

Fig. 3: Results of IR reflectography on three-year-old ink samples, normalized to Spectralon. From top to bottom: iron-gall ink, carbon ink, mixed carbon-plant ink; mixed carbon-iron-gall ink. A) image taken in visible light; B) image taken with Apollo Imaging System with SWP1250 filter; C) image taken with Apollo Imaging System with BPF1250-1510 filter; D) image taken with Apollo Imaging System with LWP1510 filter.

The lower contrast between the iron-gall ink and the writing support in the BPF1250-1510 image (Fig. 3c) compared to the higher contrast in the SWP1250 image (Fig. 3b) suggests that the iron component must have stopped absorbing light shortly above $1250 \mathrm{~nm}$; this may be due to the prevalence of insoluble black iron (III) bis-gallate in freshly made inks, which is significantly higher 
than the amount in ancient ones. ${ }^{31}$ This assumption is based on reports, from the late eighteenth century CE onwards, of originally black iron-gall inks degrading into brownish substances. ${ }^{32}$

The low contrast or 'faint trace' observed in the fresh iron-gall ink sample in the BPF1250-1510 (Fig. 3c) image can be explained by the broad spectral width of the band pass filter $(260 \mathrm{~nm})$ and the inability of the imaging sensor to discriminate the energy (wavelength) of the reflected photons. Each pixel of the InGaAs sensor simply counts and digitises the number of incoming photons that were generated by the broadband illumination using halogen lamps. For pixels corresponding to ink, the photons absorbed in the 1250-1300 nm range do not reach the sensor and, consequently, fewer total photons are counted. These pixels then have slightly lower intensity values than the pixels corresponding to the surrounding and underlying writing support, which explains the low contrast.

The spectral properties of the BPF1250-1510 filter are therefore not optimal for the unequivocal identification of the presence of iron-gall inks and carbon, but they still provide valuable information for the discrimination of inks. A series of measurements on the original documents with a device that can operate within the spectral range of 900-1700 $\mathrm{nm}$ and with a higher spectral resolution of $10 \mathrm{~nm}$ would be necessary to study and understand the absorption properties of iron-gall inks depending on their chemical composition, thickness and their interplay with the writing supports, specifically in the range of $1200-1300 \mathrm{~nm}$. A comparison of the spectral reflectance curves of iron-gall ink and the writing support would significantly narrow the range of wavelengths (down to $\pm 10 \mathrm{~nm}$ ) at which the iron-gall inks stop absorbing and lose their opacity completely.

Given the aforementioned reasons, the LWP1510 filter would be a better choice to verify the presence of carbon. This filter enables wavelengths longer than $1510 \mathrm{~nm}$ (sufficiently above the critical region of $1200-1300 \mathrm{~nm}$ ) to pass through and therefore ensures that there is only carbon absorbing in this region of the electromagnetic spectrum. However, images acquired with this filter appear dark and underexposed even under the maximum exposure time of $50 \mathrm{~ms}$ and halogen lamps set to full power. We believe that the source of this problem lies, on the one hand, in the low transmission of the filter, and on the other hand, in the apparent drop in the intensity of the light provided by the halogen lamp in this spectral region. Unfortunately, it is not possible to increase the exposure time to collect more photons. Being underexposed, the images must then undergo the post-processing step of histogram stretching in order to be

31 Krekel 1999.

32 Ribeaucourt 1797; Fuchs 2003, 160-161. 
viewed on a computer monitor by the researcher. Stretching the histogram to enhance the appearance can also create artefacts in the pictures by enhancing the noise.

Based on the outcome of the tests on ink samples prior to the imaging in Vienna and on the underexposed LWP1510 images, it was decided to select the BPF1250-1510 filter and include its results in the protocol. The permanence of iron-gall ink beyond $1250 \mathrm{~nm}$ in the tests was regarded as exceptional due to the young age of the media. We thus expected that it would still be possible to distinguish mixed inks from pure iron-gall inks, as the carbon particles dispersed in the plant or iron-gall ink base would have a stronger contrast, and therefore a much darker appearance, than the low contrast 'faint trace' of iron-gall ink.

The decision to use only one filter was additionally motivated by the need to minimise the exposure to heat generated by the broadband halogen light sources. As each scan takes ten to twenty minutes, adding a second filter to the protocol would increase the time that the fragment is exposed to heat, which is not ideal for manuscript preservation. Changes in temperature can cause the relative humidity in the area surrounding the object to fluctuate, resulting in changes to the water content of the manuscript, which desorbs water particles into the air. This desorption can create alterations, such as waves and cracks, to the writing supports; particularly susceptible are parchment, which is highly sensitive to temperature and relative humidity fluctuations, and papyrus, whose fibres are often already dry and fragile. To address this issue, we are developing a novel illumination panel set that consists of short-wave infrared (SWIR) LEDs and limits the heat emission to a technically plausible minimum. Because available LEDs emit considerably less heat than halogen or incandescent lamps, we will significantly reduce the potential risk of causing irreversible alterations to the manuscripts.

The application of this revised protocol to identify mixed inks is demonstrated by the imaging results of the bottom line of fragment A.Ch.9 (Fig. 4a). In the BPF1250-1510 image (Fig. 4b), the low contrast of this part of the text is indicative of an iron-gall ink with the exception of the last words, which show only a slight change in opacity. This confirms that the line was written using two different inks and that the last words contain carbon, as they have a strong contrast in the 1250-1510 nm range. By combining this result with the previous observations displayed in Fig. 1c, we can conclude that those words were written with a non-vitriolic carbon-iron-gall mixed ink. To confirm the identification of this ink on A.Ch.9 as a mixed carbon-iron-gall ink beyond any doubt, we should have also used the LWP1510 filter. Unfortunately, this was not done 
because we expected that the BPF1250-1510 filter alone would provide sufficient information to discriminate between iron-gall and mixed inks.

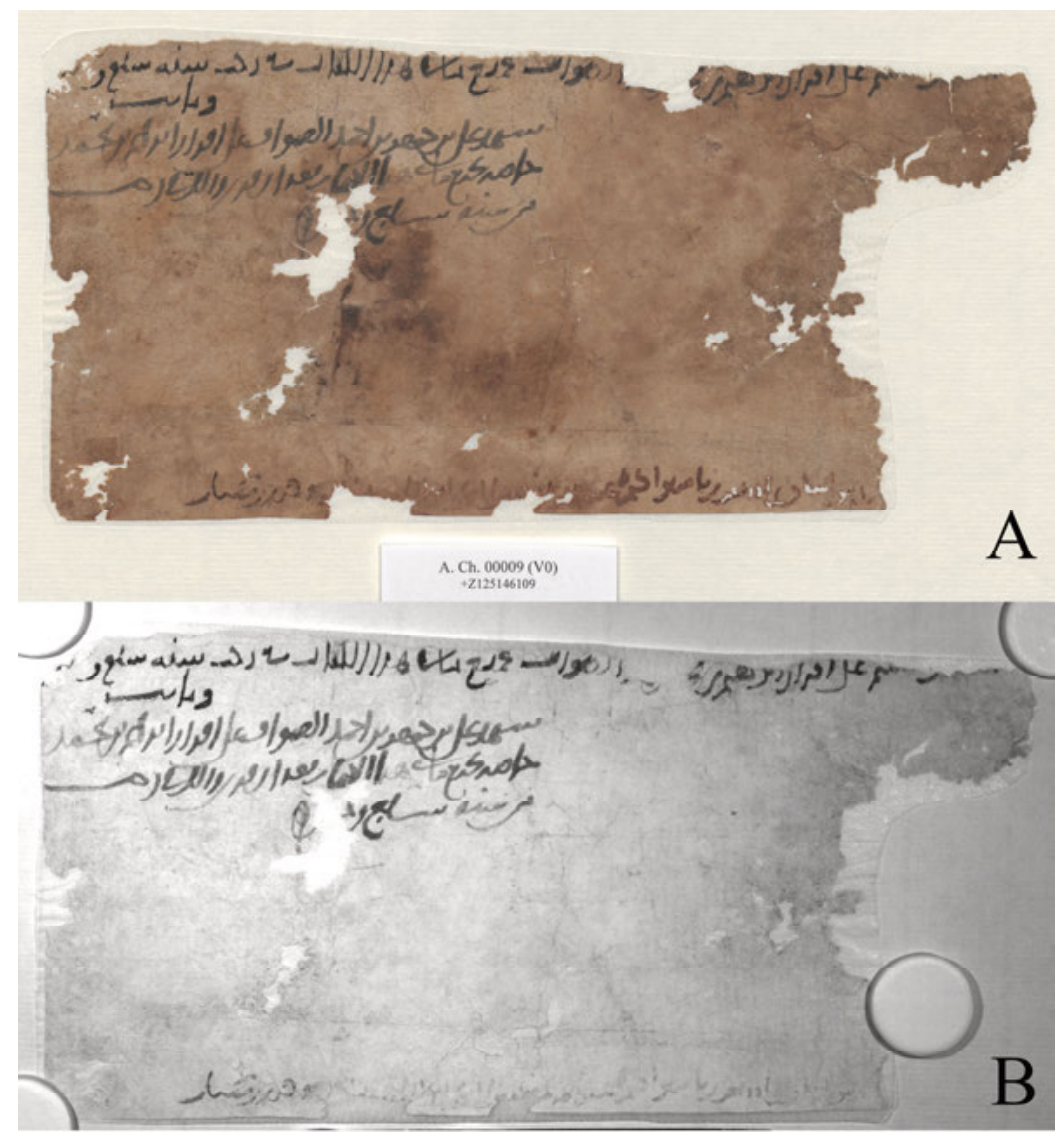

Fig. 4: A) Photograph of A.Ch.9 in visible light ( Österreichische Nationalbibliothek, Papyrussammlung; B) Picture of the same fragment taken with Apollo Imaging System with BPF12501510 filter.

Because the presence of plant and iron-gall components is determined by the absence of their signal (i.e. no contrast) above $900 \mathrm{~nm}$ and $1300 \mathrm{~nm}$, respectively, the change in opacity of a mixed ink is highly dependent on the ratio of plant or iron-gall and carbon components. When the proportion of carbon, which continues absorbing throughout the entire range, significantly outweighs that of the other ingredients, it becomes impossible to discern changes to the opacity 
of mixed inks. The exact ratio that prevents identification is not yet known, but it will be investigated in future studies.

The importance of resolving this issue becomes apparent when examining the ink used to write the tax receipt in the papyrus fragment A.P.14000 (Fig. 5), which remains difficult to identify even after performing IR reflectography.
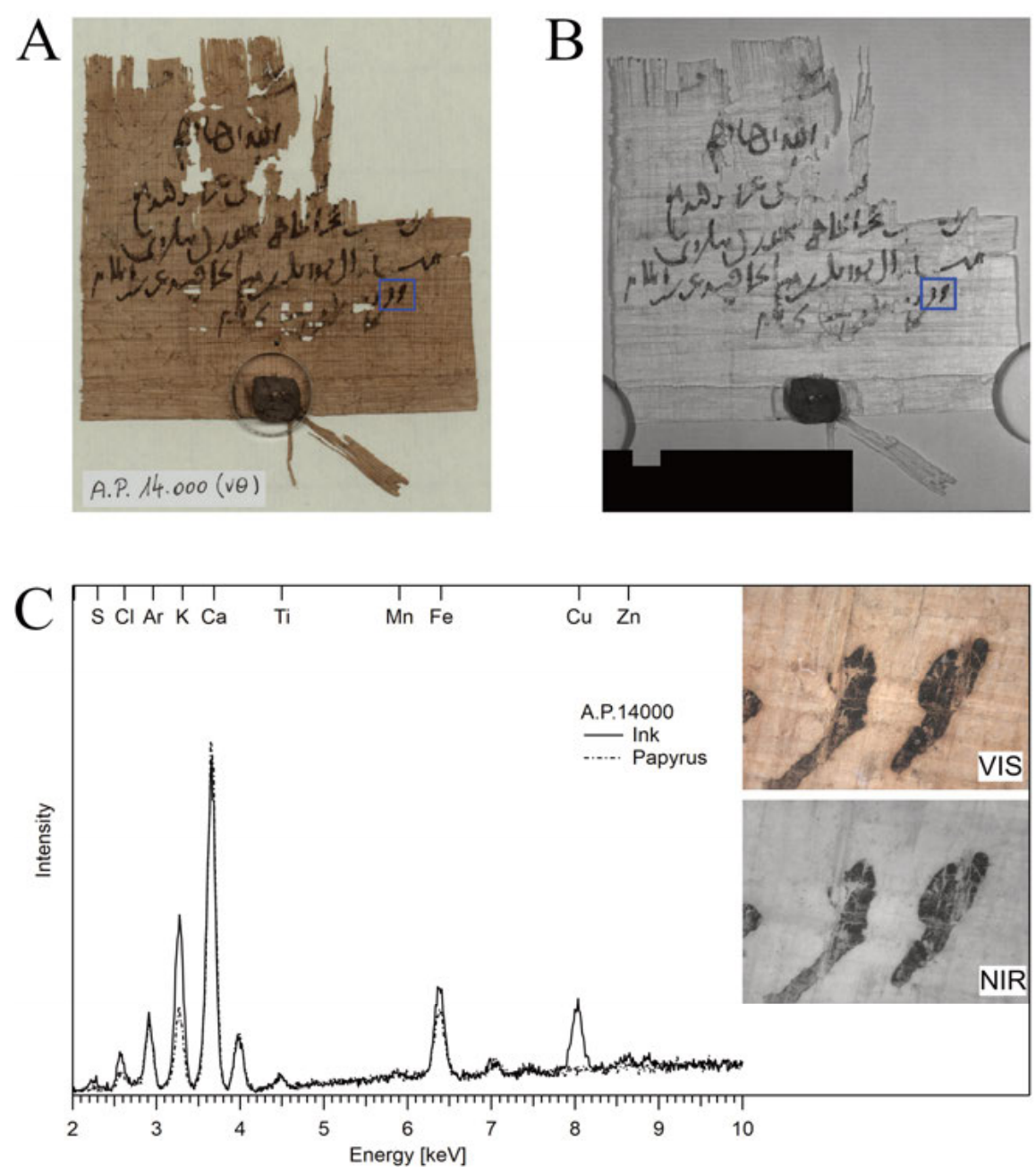

Fig. 5: A) Photograph of A.P.14000 in visible light ( Österreichische Nationalbibliothek, Papyrussammlung; B) Picture of the same fragment taken with Apollo Imaging System with BPF1250-1510 filter; C) XRF spectra of writing supports and inks, and micrographs at 50x magnification of the measured spot taken with Dino-Lite in VIS light and at $940 \mathrm{~nm}$ (NIR). 
Compared to the image taken in visible light, no change in opacity can be observed in the image taken at $940 \mathrm{~nm}$ (Fig. 5c) nor in the 1250-1510 nm range (Fig. 5b), a clear indication of a carbon-based ink. In the XRF spectra, however, there is an increase in the intensities corresponding to iron and potassium and the appearance of the copper- and zinc-related peaks in the inscribed area (black curve in Fig. 5c). These elements suggest the presence of a vitriolic component in the ink. We therefore conclude that we are dealing here, in A.P.14000, with a carbon-iron-gall mixed ink with a high carbon component.

\section{Conclusions}

In this paper we present the advantages and limitations of the improved version of the ink identification protocol, which was tested on fifteen Arabic fragments preserved in the Department of Papyri of the Austrian National Library (ÖNB), Vienna. In table 2, we provide a summary of the results obtained by the application of the improved version of the protocol on the writing media of the analysed fragments.

Table 2: Results of the analytical campaign on inks. The fragments are ordered by date.

\begin{tabular}{llll}
\hline Shelf mark & Writing & Date (CE) & Type of ink \\
\hline A.P.6004 & Receipt (v) & 900 & Carbon ink \\
& Bottom notation (v) & unknown & Carbon ink \\
& Letter (r) & unknown & Carbon ink with addition of copper \\
A.Ch.9 & First subscription & 900 , July & Carbon ink \\
& (lines 1-2) & & \\
& Second subscription & 900, July & Carbon ink \\
& (lines 3-5) & & \\
& Later remark (line 6) & After 900, July & Iron-gall ink \\
& Last words of remark & After 900, July & $\begin{array}{l}\text { Mixed ink (iron-gall with addition of } \\
\text { carbon ink) }\end{array}$ \\
& (line 6) & & $\begin{array}{l}\text { Iron-gall ink } \\
\text { A.P.4097 }\end{array}$ \\
& Protocol (r) & $902-3$ & $\begin{array}{l}\text { Mixed ink (carbon with little addition of } \\
\text { iron-gall ink) }\end{array}$ \\
& List of names (v) & After 902-3 & $\begin{array}{l}\text { Plant ink or iron-poor iron-gall ink } \\
\text { Vermillion with some red lead }\end{array}$ \\
\hline
\end{tabular}


Table 2 (continued).

\begin{tabular}{|c|c|c|c|}
\hline Shelf mark & Writing & Date (CE) & Type of ink \\
\hline A.P. 14000 & Black ink & 932 & $\begin{array}{l}\text { Mixed ink (carbon with addition of } \\
\text { vitriolic iron-gall ink) }\end{array}$ \\
\hline \multirow[t]{3}{*}{ A.Ch.7814 } & Tax receipt (v) & 937 & $\begin{array}{l}\text { Mixed ink (carbon with little addition of } \\
\text { iron-gall ink) }\end{array}$ \\
\hline & Letter (r) & $\begin{array}{l}\text { Before } 937 \\
\left(2^{\text {nd }} \text { use }\right)\end{array}$ & Carbon ink \\
\hline & Previous text (r) & $\begin{array}{l}\text { Before } 937 \\
\left(1^{\text {st }} \text { use }\right)\end{array}$ & Carbon ink \\
\hline \multirow[t]{2}{*}{ A.P.14063 } & Black ink (r) & 942, 13 Aug. & Carbon ink \\
\hline & Black ink (v) & unknown & Carbon ink \\
\hline A.Perg.82 & $\begin{array}{l}\text { Black ink (several } \\
\text { hands) }\end{array}$ & 945 & Carbon ink \\
\hline A.P. 4360 & Protocol & $944-6$ & Iron-gall ink \\
\hline A.Perg. 340 & $\begin{array}{l}\text { Brown ink (several } \\
\text { hands) }\end{array}$ & 948, Nov. & $\begin{array}{l}\text { Iron-gall ink (likely same ink for all the } \\
\text { hands) }\end{array}$ \\
\hline A.Ch.1488 & $\begin{array}{l}\text { Black-brown ink and } \\
\text { lines }\end{array}$ & $990-1$ & Iron-poor iron-gall ink \\
\hline \multirow[t]{3}{*}{ A.Ch.32363 } & Black-brown ink & $1002-3$ & Plant ink or iron-poor iron-gall ink \\
\hline & Lines & $1002-3$ & Plant ink or iron-poor iron-gall ink \\
\hline & Red inks & $1002-3$ & Iron based pigment (red ochre?) \\
\hline A.Perg. 236 & Black ink & 1002 & Carbon ink \\
\hline \multirow[t]{2}{*}{ A.Ch.7379 } & Tax receipt (r) & 1036, 8 July & Carbon ink \\
\hline & Other text (v) & $\begin{array}{l}\text { Before } 1036 \\
\left(1^{\text {st }} \text { use) }\right.\end{array}$ & Carbon ink \\
\hline \multirow{3}{*}{$\begin{array}{l}\text { A.Ch. } 1252+ \\
\text { A.Ch.14324 }\end{array}$} & Black ink & $1044-5$ & Iron-gall ink \\
\hline & Lines & $1044-5$ & Iron-gall ink \\
\hline & Red ink & $1044-5$ & Vermillion with some red lead \\
\hline
\end{tabular}

This study has shown that adding an IRR mobile camera to the standard protocol would enable the detection of carbon in the inks, and thus the discrimination between iron-gall and mixed inks. For the unequivocal identification of carbon, the IRR investigation needs to be conducted at wavelengths higher than $1300 \mathrm{~nm}$. For this reason, the Long Wave Pass Filter (LWP1510) will replace the initially selected Band Pass Filter (BPF1250-1510) in our routine protocol. To address the issue of LWP1510 images being underexposed and reduce the risk of 
introducing post-processing artefacts, we will work closely with the manufacturers of the system to design a new, LED-based illumination set that is optimised for the range of 1500-1700 nm. LED light sources, which irradiate less heat, will also render the ink identification protocol much safer for the objects from a conservation perspective.

\section{Acknowledgements}

We would like to thank the director of the Vienna Papyri Collection, Bertrand Palme, and the staff at the National Library of Austria (ÖNB) for their help and support, especially Simone Suppan and Andrea Donau. Our special thanks go to the people at OPUS Instruments, who provided immediate answers to our questions and helped us to get acquainted with the system. Our warmest gratitude goes also to our research team in Berlin and Hamburg, in particular to Olivier Bonnerot, Sebastian Bosch, Zina Cohen, Tea Ghigo and Greg Nehring.

The research for this paper was funded by the Deutsche Forschungsgemeinschaft (DFG, German Research Foundation) under Germany’s Excellence Strategy - EXC 2176 'Understanding Written Artefacts: Material, Interaction and Transmission in Manuscript Cultures', project no. 390893796. The research was conducted within the scope of the Centre for the Study of Manuscript Cultures (CSMC) at Hamburg University.

\section{References}

Bausi, Alessandro, Pier Giorgio Borbone, Françoise Briquel-Chatonnet, Paola Buzi, Jost Gippert, Caroline Macé, Marilena Maniaci, Zisis Melissakēs, Laura E. Parodi and Witold Witakowski (eds), with Eugenia Sokolinski (2015), Comparative Oriental Manuscript Studies: An Introduction, Hamburg: COMSt.

Brun, Emmanuel, Marine Cotte, Jonathan Wright, Marie Ruat, Pieter Tack, Lazlo Vincze, Claudio Ferrero, Daniel Delattre and Vito Mocella (2016), 'Revealing metallic ink in Herculaneum papyri', Proceedings of the National Academy of Science, 113: 3751-3754.

Christiansen, Thomas, Marine Cotte, René Loredo-Portales, Poul Erik Lindelof, Kell Mortensen, Kim Ryholt and Sine Larsen (2017), 'The nature of ancient Egyptian copper containing carbon inks is revealed by synchrotron radiation based X-ray microscopy', Scientific Report, 7:15346 (1-8) <doi.org/10.1038/s41598-017-15652-7>.

Christiansen, Thomas, Marine Cotte, Wout de Nolf, Elouan Mouro, Juan Reyes-Herrera, Steven de Meyer, Frederik Vanmeert, Nati Salvadó, Victor Gonzalez, Poul Erik Lindelof, Kell Mortensen, Kim Ryholt, Koen Janssens and Sine Larsen (2020), 'Insights into the composition of ancient Egyptian red and black inks on papyri achieved by synchrotron-based microanalyses', Proceedings of the National Academy of Science, 117/45: 27825-27835. 
Cohen, Zina (2020), Composition analysis of writing materials in Genizah Documents, PhD dissertation, Paris Sciences \& Lettres (École Pratique des Hautes Études) and Hamburg University.

Cohen, Zina, Judith Olszowy-Schlanger, Oliver Hahn and Ira Rabin (2017) 'Composition Analysis of Writing Materials in Geniza Fragments', in Wandrey (ed.) 2017, 323-338.

Colini, Claudia (2018), From Recipes to Material Analysis: the Arabic tradition of Black Inks and Paper Coatings ( $9^{\text {th }}$ to $20^{\text {th }}$ century), PhD dissertation, Hamburg University.

Colini, Claudia, Oliver Hahn, Olivier Bonnerot, Simon Steger, Zina Cohen, Tea Ghigo, Thomas Christiansen, Marina Bicchieri, Paola Biocca, Myriam Krutzsch, and Ira Rabin (2018), 'The quest for the mixed inks', manuscript cultures, 11: 41-49.

Fuchs, Robert (2003), 'The history of chemical reinforcement of texts in manuscript - What should we do now', in Gillian Fellows-Jensen and Peter Springborg (eds), Care and Conservation of Manuscripts 7, Proceedings of the Seventh International Seminar Held at the Royal Library, Copenhagen 18th-19th April 2002, Copenhagen: Museum Tusculanum Press, 159-170.

Geissbühler, Mirjam, Georg Dietz, Oliver Hahn and Ira Rabin (2018), 'Advanced codicological studies of Cod. Germ. (Hamburg, Staats- und Universitätsbibliothek): Part 2', manuscript cultures, 11: 133-139.

Ghigo, Tea (2020), A Systematic Scientific Study of Coptic Inks from the Late Roman Period to the Middle Ages, PhD dissertation, Hamburg University and La Sapienza (Rome) University.

Ghigo, Tea, Ira Rabin and Paola Buzi (2020), 'Black Egyptian inks in Late Antiquity: new insights on their manufacture and use', Archaeological and Anthropological Sciences, 12:70 (1-14) <doi.org/10.1007/s12520-019-00977-3>.

Grob, Eva Mira (2010), Documentary Arabic private and business letters on papyrus: form and function, content and context (Archiv für Papyrusforschung und verwandte Gebiete. Beihefte, 29), Berlin: De Gruyter.

Grohmann, Adolf (1952), From the world of Arabic papyri, Cairo: Al-Maaref Press.

Hahn, Oliver, Wolfgang Malzer, Birgit Kanngießer and Burkhard Beckhoff (2004), 'Characterization of iron-gall inks in historical manuscripts and music compositions using $\mathrm{x}$-ray fluorescence spectrometry', X-Ray Spectrometry, 33: 234-239.

Hahn, Oliver, Timo Wolff, Hartmut-Ortwien Feistel, Ira Rabin and Malachi Beit-Arié (2008a), 'The Erfurt Hebrew Giant Bible and the Experimental XRF Analysis of Ink and Plummet Composition', Gazette du Livre Médiéval, 51: 16-29.

Hahn, Oliver, Max Wilke and Timo Wolff (2008b), 'Influence of aqueous calcium phytate/ calcium hydrogen carbonate treatment on the chemical composition of iron gall inks', Restaurator, 29/4: 235-250.

Haim, Ofir, Michael Shenkar and Sharof Kurbanov (2016), 'The Earliest Arabic manuscripts written on paper: Three letters from Sanjar-Shah (Tajikistan)', Jerusalem Studies in Arabic and Islam, 43: 141-189.

Krekel, Christoph (1999) 'The chemistry of historical iron gall inks: Understanding the chemistry of writing inks used to prepare historical documents', International journal of forensic document examiners, 5: 54-58.

Mrusek, Ralf, Robert Fuchs and Doris Oltrogge (1995), 'Spektrale Fenster zur Vergangenheit. Ein neues Reflektographieverfahren zur Untersuchung von Buchmalerei und historischem Schriftgut', Naturwissenschaften, 82: 68-79. 
Nehring Grzegorz, Olivier Bonnerot, Marius Gerhardt, Myriam Krutzsch and Ira Rabin (2021), 'Looking for the missing link in the evolution of black inks', Archaeological and Anthropological Sciences, 13:71 (1-10) <doi.org/10.1007/s12520-021-01320-5>.

Nir-El, Yoram and Magen Broshi (1996) 'The black ink of the Qumran scrolls', Dead Sea Discoveries, 3/2: 157-167.

Rabin, Ira (2015), 'Instrumental analysis in manuscript studies', in Bausi et al. (eds) 2015, 27-30.

Rabin, Ira (2017), 'Building a Bridge from the Dead Sea Scrolls to Mediaeval Hebrew Manuscripts', in Wandrey (ed.) 2017, 309-322.

Rabin, Ira, Oliver Hahn, Roger Easton Jr., Keith T. Knox, Ken Boydston, Gregory Heyworth, Timoty Leonardi and Michael Phelps (2015), 'Initial inspection of reagent damage to the Vercelli Book', Comparative Oriental Manuscript Studies Bulletin, 1/1: 34-45.

Rabin, Ira, Roman Schütz, Anka Kohl, Timo Wolff, Roald Tagle, Simone Pentzien, Oliver Hahn and Stephen Emmel (2012), 'Identification and classification of historical writing inks in spectroscopy: a methodological overview', Comparative Oriental Manuscript Studies Newsletter, 3: 26-30.

Rabin, Ira, Carsten Wintermann and Oliver Hahn (2019), 'Ink characterization, performed in Biblioteca Medicea Laurenziana (September 2018)', Analecta Papyrologica, 31: 301-313.

Ribeaucourt (1797), 'Über die gewöhnliche schwarze Tinte', Crells chemische Annalen, 28: 41-54.

Rustow, Marina (2020), The Lost Archive: Traces of a Caliphate in a Cairo Synagogue, Princeton: University Press.

Shatzmiller, Maya (2018), 'The adoption of paper in the Middle East, 700-1300 AD', Journal of the Economic and Social History of the Orient, 61: 461-490.

Wandrey, Irina (ed.) (2017), Jewish Manuscript Cultures. New Perspectives (Studies in Manuscript Cultures, 13), Berlin: De Gruyter.

Yussef-Grob, Eva Mira (2015), 'The earliest paper documents in the Vienna collection revisited', Chronique d'Égypte, 90: 431-443.

Zerdoun Bat-Yehouda, Monique (1983), Les encres noires au Moyen Âge (jusqu'à 1600), Paris: CNRS Éditions [reprint: 2003]. 
